Original

\title{
Expression of cytokeratins 10, 13, 14 and 19 in oral lichen planus
}

\author{
Claudio M. C. Jacques, Ana L. C. Pereira, Vivian Maia, Tullia Cuzzi \\ and Marcia Ramos-e-Silva \\ Oral Dermatology Out-Patient Clinic, Sector of Dermatology and Post-Graduation Course, HUCFF-UFRJ, \\ and School of Medicine, Federal University of Rio de Janeiro, Brazil
}

(Received 23 April 2008 and accepted 15 April 2009)

\begin{abstract}
OLP is a chronic inflammatory disease of unknown etiology that may develop into squamouscell carcinoma. Cytokeratins, which are important components of the cytoskeleton, are excellent epithelial differentiation markers used to study neoplastic and inflammatory diseases. To study the profile of cytokeratins in OLP and their possible association with dysplastic alterations, monoclonal antibodies were used for cytokeratins 10, 13, 14 and 19, in 26 samples of OLP. The streptavidin-biotin technique was employed in paraffin-embedded tissue sections. Sample analysis revealed suprabasal expression of cytokeratin 10 in 16/17 samples, 14 of them with reduced expression; suprabasal expression of cytokeratin 13 in 18/23, 16 of them with delay; basal and suprabasal expression of cytokeratin 14 in all samples; and focal basal expression of cytokeratin 19 in 4/21. Expression of cytokeratins 10,13 and 14 was altered in OLP lesions. The inflammatory process and hyperkeratosis or parakeratosis seem to have interfered with the expression of these CKs. Cytokeratin 19 was expressed in the lesions, in a pattern similar to that mentioned in the literature for the non-keratinized oral mucosa. The presence of mild dysplasia did not change the expression of the cytokeratins studied. No differences in pattern of expression were observed between keratinized and non-keratinized areas in the lesions caused by OLP. (J Oral Sci 51, 355-365, 2009)
\end{abstract}

Correspondence to Dr. Marcia Ramos-e-Silva, Rua Dona Mariana 143 / C-32, Botafogo 22280-020, Rio de Janeiro Brazil

Tel: +55-21-22864632

Fax: +55-21-22864632

E-mail: ramos.e.silva@dermato.med.br
Keywords: lichen planus; oral; cytokeratins; mouth.

\section{Introduction}

Lichen Planus (LP) is an inflammatory dermatosis, first described by Erasmus Wilson in 1869. Its etiology remains unknown, although there are strong indications that its physiopathogenic mechanism is autoimmune. The disease can affect any part of the oral cavity; however the lesions are frequently detected in the buccal mucosa. Different from cutaneous lesions, oral lichen planus (OLP) affects individuals usually above age 40 and presents a chronic evolution that can persist for over twenty years (1). It may provoke pain or burning sensation, and can exhibit malignant transformation (2).

The World Health Organization (WHO) recognizes seven different clinical forms of OLP: reticular, plaquelike, papular, erosive, atrophic, bullous, and ulcerated (3).

The diagnosis is based on the clinical findings, with confirmation through histopathological examination. However, there are many situations where the diagnosis is doubtful, due to the fact that the clinical-histopathological alterations could be shared by other diseases, as for instance the chronic form of the disease graft-versus-host of the oral mucosa $(4,5)$.

Cytokeratins (CKs) are intracytoplasmatic protein filaments, so named by cellular biologists and pathologists to describe the keratins found in the cytoplasm of the epithelial cells, aiming at distinguishing them from specialized keratins, as those that compose nails and hair (6). In the cytoplasm of eukaryotic cells, CKs are an important component of the cytoskeleton, being responsible for the function of structural maintenance of the cell and 
tissue, protecting against mechanical traumas, and also having a probable role in the intercommunication among adjacent cells $(7,8)$.

Due to the great stability and little variability of CKs in the tissue, studies of primary or secondary alterations of those proteins in the tumorous and inflammatory dermatological diseases are frequent, and attempt to understand better the etiopathogenesis of those diseases, as well as their application for improving dermatological diagnosis (9).

\section{Specimen}

\section{Methods}

Twenty-six samples of OLP lesions obtained from different individuals were selected for this study. Of these, twenty-three were from the collection of the Sector of Pathology of University Hospital of the Federal University of Rio de Janeiro, and three samples were from a private clinic. This material was selected based on the examination of the samples stained by hematoxylin-eosin, for confirmation of the diagnosis. The diagnosis of OLP was based on the following five histopathological alterations: dyskeratosis (hyperkeratosis and/or parakeratosis), vacuolation of the basal layer, band-like inflammatory infiltrate in the superficial dermis, keratinocyte necrosis and pigmentary incontinence. It is opportune to stress that special emphasis was given to the association of at least two of the first three above-described aspects.

Also, presence or absence of keratinocytes with dysplastic alterations in these samples was recorded, as dysplastic alterations were considered as a parameter listed by the Collaborating Center with the World Health Organization for Precancerous Oral Lesions (10). In relation to the grade of dysplasia, the presence of one or two of the abovementioned characteristics was considered as "light" grade, presence of three or four characteristics as "moderate" grade, and presence of more than four characteristics as "severe" dysplasia, as published in 1976 by Bánóczy \& Csiba (11). As shown in Table 1, 11 of the 26 samples studied presented dysplastic alterations, corresponding to $42.3 \%$. In all of the cases, the dysplastic alterations were considered of light grade and focal character, except for sample numbers 7, 16 and 20 which presented these alterations diffusely throughout the epithelium.

A history of bone marrow transplant was used as exclusion criterion, due to the difficulty in the differential diagnosis between OLP and the graft-versus-host chronic disease of the oral mucosa (5).

Data were collected regarding sex, age, clinical forms, and location of the lesions; through anamnesis and clinical
Table 1 Clinical-epidemiological data and dysplasia of samples studied

\begin{tabular}{|c|c|c|c|c|c|}
\hline Sample \# & Sex & Age & Site & Clinical Form & Dysplasia \\
\hline 1 & $F$ & 86 & $\mathrm{BM}$ & ERO & + (mild and focal) \\
\hline 2 & $\mathbf{F}$ & 60 & $\mathrm{BM}$ & RET & + (mild and focal) \\
\hline 3 & F & 33 & $\mathrm{BM}$ & ERO & + (mild and focal) \\
\hline 4 & $F$ & 35 & LIP & RET & - \\
\hline 5 & F & 64 & $\mathrm{BM}$ & PLA & $+($ mild and focal) \\
\hline 6 & $\mathrm{~F}$ & 42 & $\mathrm{BM}$ & RET & - \\
\hline 7 & $\mathbf{F}$ & 71 & $\mathrm{BM}$ & PLA & $+($ mild and diffuse) \\
\hline 8 & $\mathbf{F}$ & 51 & $\mathrm{BM}$ & PLA & - \\
\hline 9 & M & 69 & LIP & RET & $+($ mild and focal $)$ \\
\hline 10 & $F$ & 45 & $\mathrm{BM}$ & RET & - \\
\hline 11 & $F$ & 62 & $\mathrm{BM}$ & ERO & - \\
\hline 12 & $\mathrm{M}$ & 57 & $\mathrm{BM}$ & ERO & - \\
\hline 13 & $\mathrm{~F}$ & 67 & LIP & RET & - \\
\hline 14 & $\mathrm{~F}$ & 34 & $\mathrm{BM}$ & PLA & $+($ mild and focal $)$ \\
\hline 15 & F & 54 & DT & PLA & + (mild and focal) \\
\hline 16 & $\mathrm{~F}$ & 62 & $\mathrm{BM}$ & PLA & + (mild and diffuse) \\
\hline 17 & $\mathrm{~F}$ & 65 & $\mathrm{BM}$ & PLA & - \\
\hline 18 & $\mathbf{F}$ & 25 & $\mathrm{BM}$ & RET & - \\
\hline 19 & $\mathrm{~F}$ & 57 & G & ATR & - \\
\hline 20 & $\mathrm{~F}$ & 76 & $\mathrm{BM}$ & PLA & + (mild and diffuse) \\
\hline 21 & $F$ & 59 & G & ERO & - \\
\hline 22 & $F$ & 72 & $\mathrm{BM}$ & PLA & $+($ mild and focal $)$ \\
\hline 23 & $\mathbf{F}$ & 51 & $\mathrm{BM}$ & RET & - \\
\hline 24 & $F$ & 70 & $\mathrm{BM}$ & RET & - \\
\hline 25 & $\mathbf{F}$ & 50 & $\mathrm{BM}$ & PLA & - \\
\hline 26 & F & 59 & $\mathrm{BM}$ & PAP & - \\
\hline
\end{tabular}

M: male; F: female; BM: buccal mucosa; LIP: lip; G: gengiva; DT: dorsum of tongue; RET: reticular; PLA: plaque-type; ERO: erosive; ATR: atrophic; PAP: papular; $(+)$ positive; $(-)$ negative

examination, or from the patient's medical records, in the cases where it was not possible to interview and to examine the patients (Table 1). Of the 26 samples examined, 24 $(92.3 \%)$ were from female individuals, while the masculine sex was represented by 2 samples $(7.7 \%)$. Six individuals were below 50,8 between 50 and 59,7 between 60 and 69 and 5 individuals of age 70 or above. Most of the lesions biopsied were located in the buccal mucosa (20), corresponding to $77 \%$ of the samples. Of the remaining six, two were located in the gingiva (7.7\%), three in lips (11.5\%) and one on the back of the tongue (3.8\%).

In relation to the clinical form, nine of the lesions biopsied (34.7\%) corresponded to the reticular form, ten $(38.5 \%)$ to the plaque form, and five $(19.2 \%)$ to the erosive form. Lesions of the papulous and atrophic forms were biopsied in one patient each, corresponding to a percentage 
of $3.8 \%$ for each one.

\section{Immunohistochemical staining}

The expression of CKs 10,13,14 and 19 was studied by the streptavidin-biotin technique. Table 2 presents the primary antibodies used, as well as their respective manufacturers and dilutions. The incubation period for all antibodies was 12 hours. The tissue samples of the oral cavity were obtained by punch biopsy, fixed in formaldehyde buffered at $10 \%$ and blocked in paraffin. The Novostain Universal Detection Kit was used (NOVOCASTRA NCL-RTU-D - Newcastle Upon Tyne, UK). The positivity of the reaction was considered based on the clear marking of the material, also observing parameters regarding its location within the epithelium (basal and/or suprabasal),

Table 2 Primary antibodies used

\begin{tabular}{cccc}
\hline CKs & Code & Manufacturer & Dilution \\
\hline 10 & M7002 & DAKO & $1 / 50$ \\
13 & M7003 & DAKO & $1 / 25$ \\
14 & NCL-LL002 & NOVOCASTRA & $1 / 20$ \\
19 & NCL-CK19 & NOVOCASTRA & $1 / 100$ \\
\hline
\end{tabular}

the distribution of the markings (diffuse or focal), and presence or not of delay in the marking. A marking present in the entire epithelial extension was defined as diffuse and a marking observed in some segments of the epithelium as focal. A delay was considered in the marking, when this was evidenced in the suprabasal portion, with negatively stained basal and parabasal cells.

\section{Results}

To accomplish the objectives proposed for the study, statistical analysis of the data obtained would be required. However, due to the low variation in frequency, it was not possible to subject the data regarding the four CKs to statistical analysis, when comparing its expression in the normal mucosa.

\section{Cytokeratins}

For technical reasons, it was not possible to perform immunohistochemical reactions for four CKs in each one of the twenty-six samples. The results obtained separately for each CK are presented below.

\section{Cytokeratin 10}

Reactions were accomplished for CK 10 in 17 samples,

Table 3 Distribution of CKs in samples studied

\begin{tabular}{|c|c|c|c|c|c|c|c|c|}
\hline Sample \# & \multicolumn{2}{|c|}{ CK10 } & \multicolumn{2}{|r|}{ CK13 } & \multicolumn{2}{|r|}{ CK14 } & \multicolumn{2}{|c|}{ CK19 } \\
\hline & $\mathrm{B}$ & SB & $\mathrm{B}$ & $\mathrm{SB}$ & $\mathrm{B}$ & $\mathrm{SB}$ & $\mathrm{B}$ & $\mathrm{SB}$ \\
\hline 1 & - & $+($ focal $)$ & - & + (with delay) & + & + & - & - \\
\hline 2 & - & $+($ focal $)$ & $\mathrm{x}$ & $X$ & + & + & $\mathrm{X}$ & $X$ \\
\hline 3 & - & $+($ focal $)$ & - & + (with delay) & + & + & - & - \\
\hline 4 & - & $+($ focal $)$ & - & - & + & + & - & - \\
\hline 5 & - & $+($ focal $)$ & - & + (with delay) & + & + & - & - \\
\hline 6 & - & $+($ focal $)$ & - & $+($ with delay $)$ & + & + & - & - \\
\hline 7 & - & - & - & - & + & + & $\mathrm{X}$ & $\mathrm{X}$ \\
\hline 8 & - & $+($ focal $)$ & - & + & + & $+($ parabasal, focal $)$ & - & - \\
\hline 9 & - & $+($ focal $)$ & - & $+($ with delay) & $\mathrm{X}$ & X & $\mathrm{X}$ & $\mathrm{X}$ \\
\hline 10 & - & $+($ focal $)$ & - & $+($ with delay $)$ & $\mathrm{X}$ & $X$ & - & - \\
\hline 11 & - & $+($ focal $)$ & - & $+($ with delay $)$ & + & + & - & - \\
\hline 12 & - & $+($ focal $)$ & - & + (with delay) & + & + & $+($ focal $)$ & - \\
\hline 13 & - & + & - & + & + & + & - & - \\
\hline 14 & - & + & - & + (with delay) & + & + & - & - \\
\hline 15 & - & $+($ focal $)$ & - & - & + & + & - & - \\
\hline 16 & - & $+($ focal $)$ & $\mathrm{X}$ & $\mathrm{X}$ & $\mathrm{X}$ & $\mathrm{x}$ & $+($ focal $)$ & - \\
\hline 17 & - & $+($ focal $)$ & - & + (with focal delay) & + & + & - & - \\
\hline 18 & $\mathrm{X}$ & $X$ & - & + (with delay) & + & + & $+($ focal $)$ & - \\
\hline 19 & $\mathrm{X}$ & $\mathrm{X}$ & - & + (with focal delay) & + & + & $+($ focal $)$ & - \\
\hline 20 & $\mathrm{X}$ & $\mathrm{X}$ & - & - & + & + & - & - \\
\hline 21 & $\mathrm{X}$ & $\mathrm{X}$ & - & + (with focal delay) & + & + & - & - \\
\hline 22 & $\mathrm{X}$ & $\mathrm{X}$ & _ & - & + & + & - & - \\
\hline 23 & $\mathrm{X}$ & $\mathrm{X}$ & - & + (with delay) & + & + & $\mathrm{X}$ & $\mathrm{X}$ \\
\hline 24 & $\mathrm{X}$ & $\mathrm{X}$ & - & + (with delay) & + & + & $\mathrm{X}$ & $\mathrm{X}$ \\
\hline 25 & $\mathrm{X}$ & $\mathrm{X}$ & - & + (with delay) & + & + & - & - \\
\hline 26 & $\mathrm{X}$ & $X$ & $\mathrm{X}$ & $\mathrm{X}$ & + & + & - & - \\
\hline
\end{tabular}

B: basal marking; SB: suprabasal marking; $(+)$ positive; (-) negative; X: not performed 


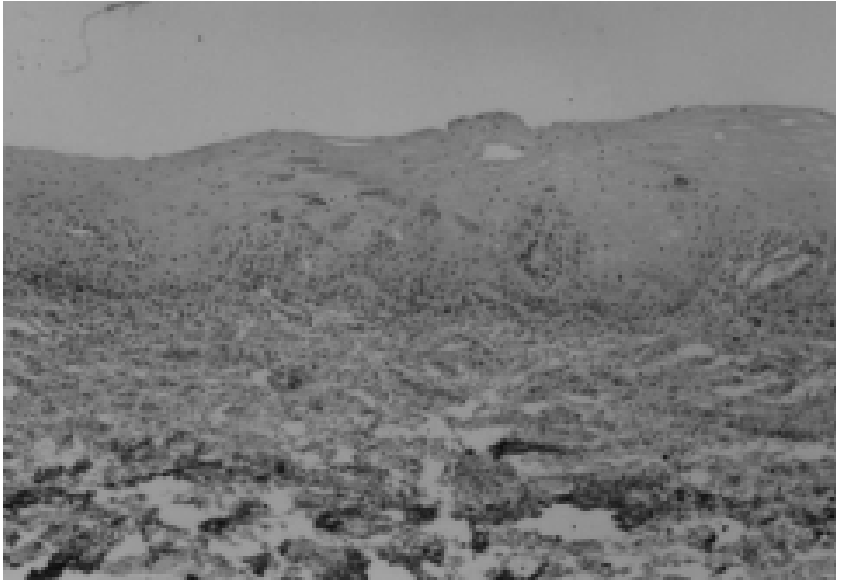

Fig. 1 CK 10: marking restricted to few suprabasal cells in non-keratinized mucosal lesion. Obj.: $\times 10$.

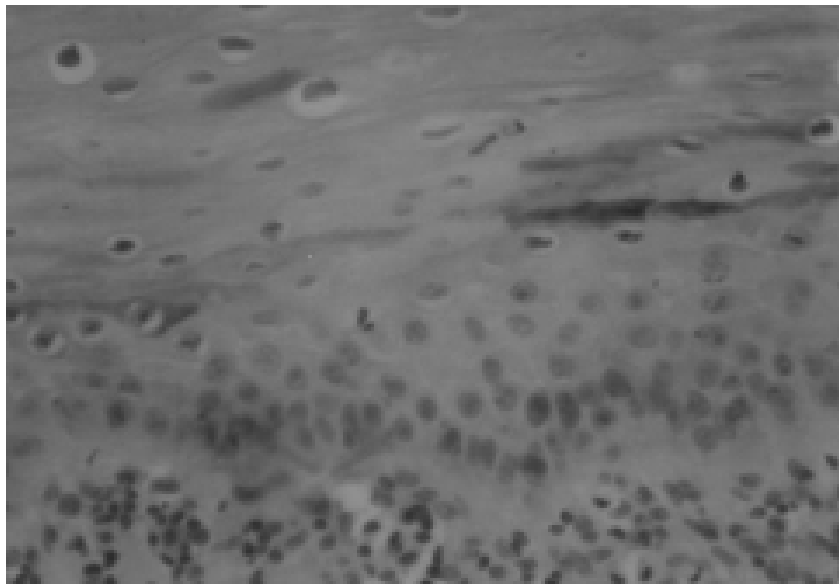

Fig. 2 CK 10: focal suprabasal marking in non-keratinized mucosa. Obj: $\times 40$

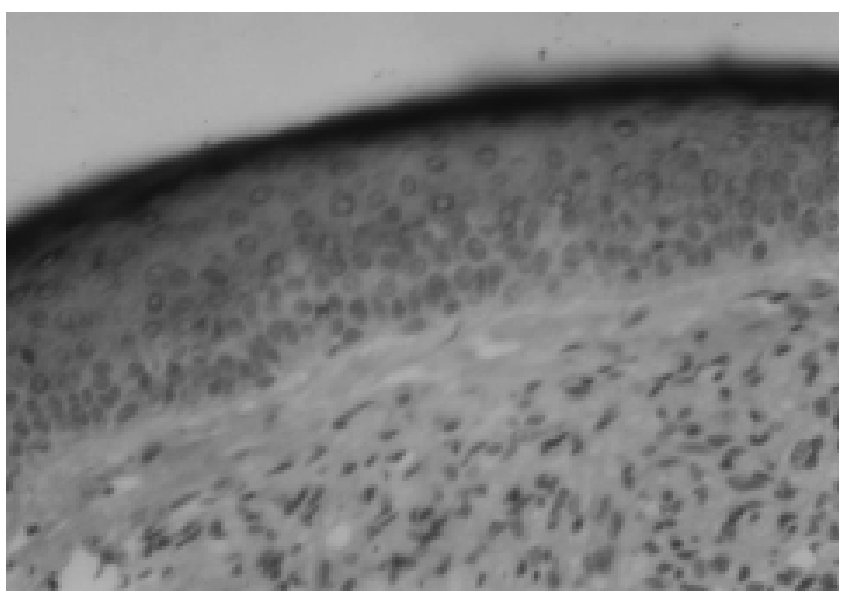

Fig. 3 CK 10: diffuse suprabasal marking. Obj.: $\times 10$

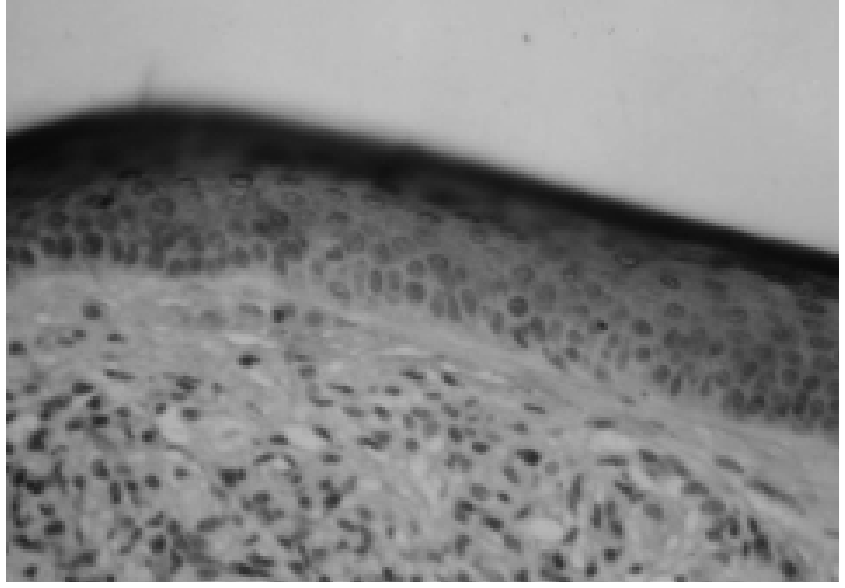

Fig. 4 CK 10: same. Obj.: $\times 10$

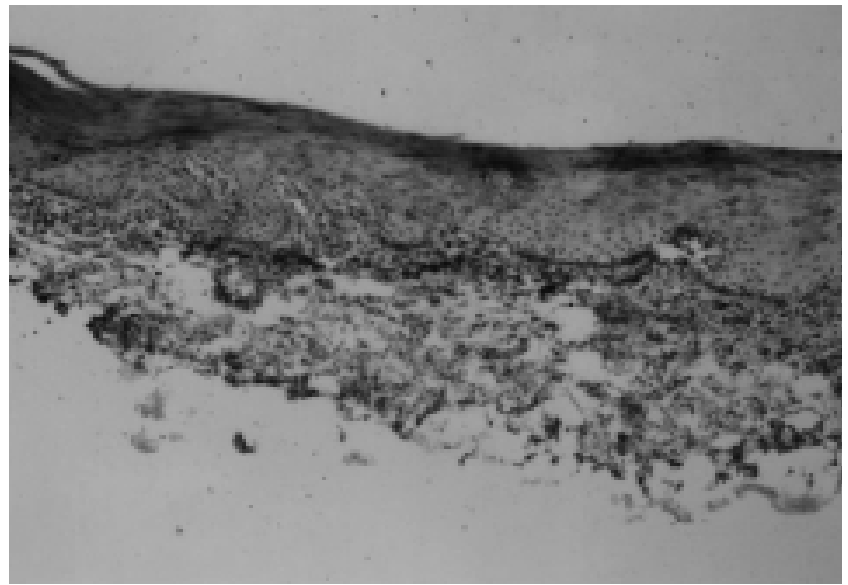

Fig. 5 CK 10: positive marking in some suprabasal cells. Obj.: $\times 10$

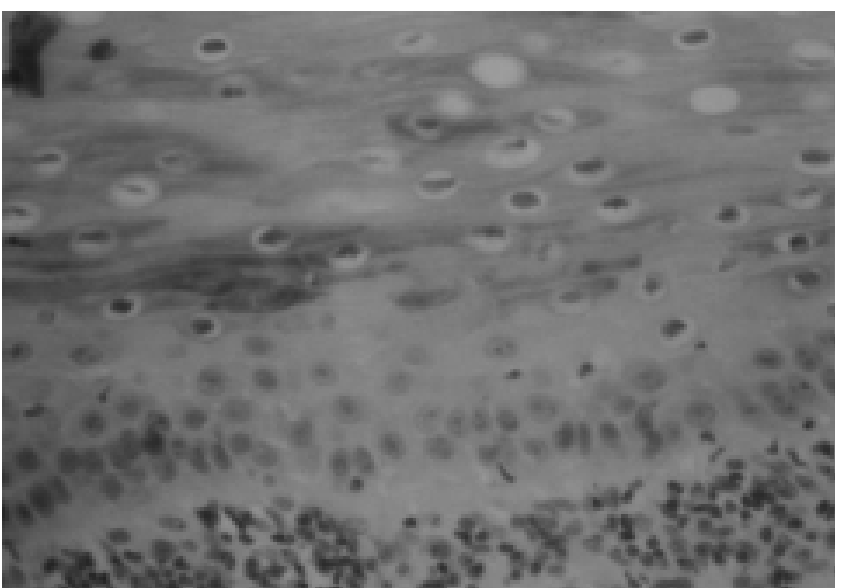

Fig. 6 CK 10: same. Obj.: $\times 40$ 
with results obtained listed in Table 3. In all of the samples, except one (sample number 7), positive marking was observed along the depth of the Malpighi stratum (Figs. 1 and 2). In two of these 16 positive samples, marking was diffuse (Figs. 3, 4). In the other 14 samples, marking was considered focal (Figs. 5 and 6). Of these, nine cases were obtained in which marking shown was quite discrete. In none of the samples, positive marking along the basal layer was noticed.

\section{Cytokeratin 13}

Reactions were accomplished with antibodies for CK 13 in 23 tissue samples. The results obtained can be found in Table 3. All 23 samples presented negative marking in the basal layer. As for the suprabasal portion of the epithelium, five of the 23 samples (21.7\%) presented negative marking, and $18(78.3 \%)$ presented positive marking. Among the latter, 16 presented delay in the marking (88.8\%) (Figs. 7-9).

\section{Cytokeratin 14}

Twenty-three samples were studied regarding CK 14. The results obtained are shown in Table 3. The marking for CK 14 was positive in 100\% of the studied samples. In two samples, the marking was limited to the basal layer and parabasal portion of the epithelium (Figs. 11, 13 and 15). In the remaining samples, the marking included the totality of the basal and Malpighi layers (Figs. 10, 12 and 14). In 20 samples, the alteration in the marking pattern was diffuse. In the remaining 3 samples, the marking pattern was considered focal.

Table 4 Distribution of cytokeratins in normal oral mucosa epithelium

\begin{tabular}{ccc}
\hline & Basal layer & Suprabasal layer \\
\hline \multirow{2}{*}{ Non-keratinized mucosa } & CKs 5 and 14 & \\
& CK 19 & CKs 4 and 13 \\
(focal and discrete) & \\
Keratinized mucosa & CKs 5 and 14 & CKs 1,2,10 and 11 \\
\hline
\end{tabular}

\section{Cytokeratin 19}

Twenty-one samples were studied for CK 19 (Table 3). Only 4 samples (19\%) presented marking in the basal layer, all presenting focal and discrete character (Figs. 16-19). All 21 samples showed negative marking in the suprabasal portion of the epidermis.

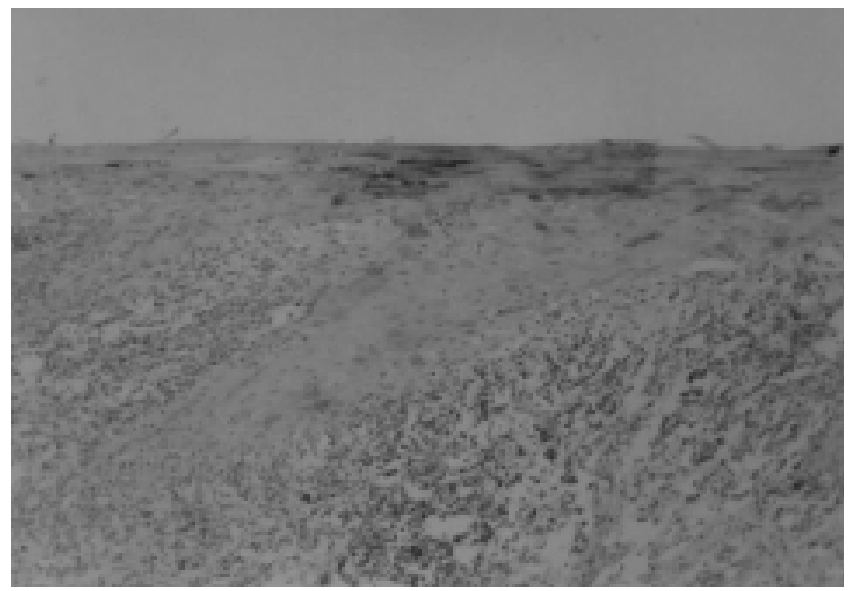

Fig. 7 CK 13: marking of the upper portions of the Malpighian layer, with delay. Obj: $\times 10$

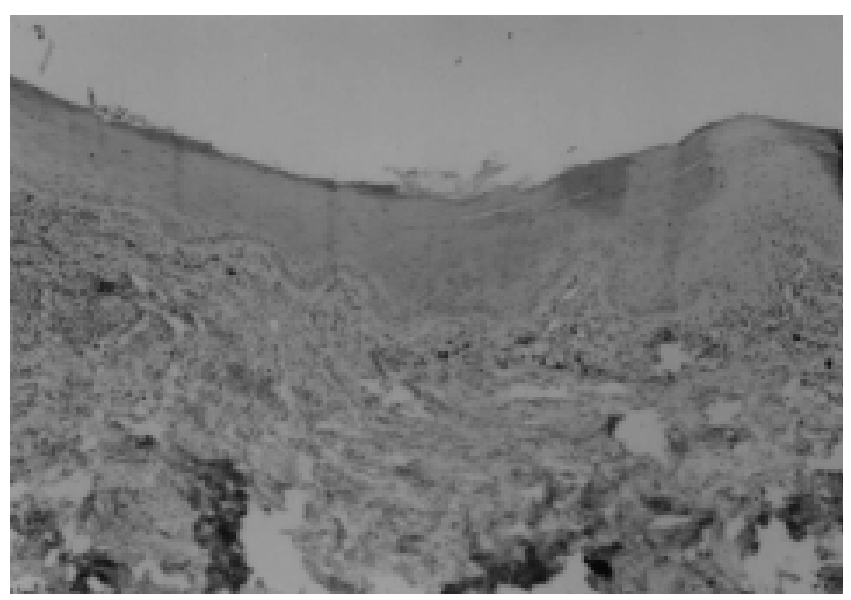

Fig. 8 CK 13: delay in the expression with negative marking, on basal and parabasal cells. Obj.: $\times 10$

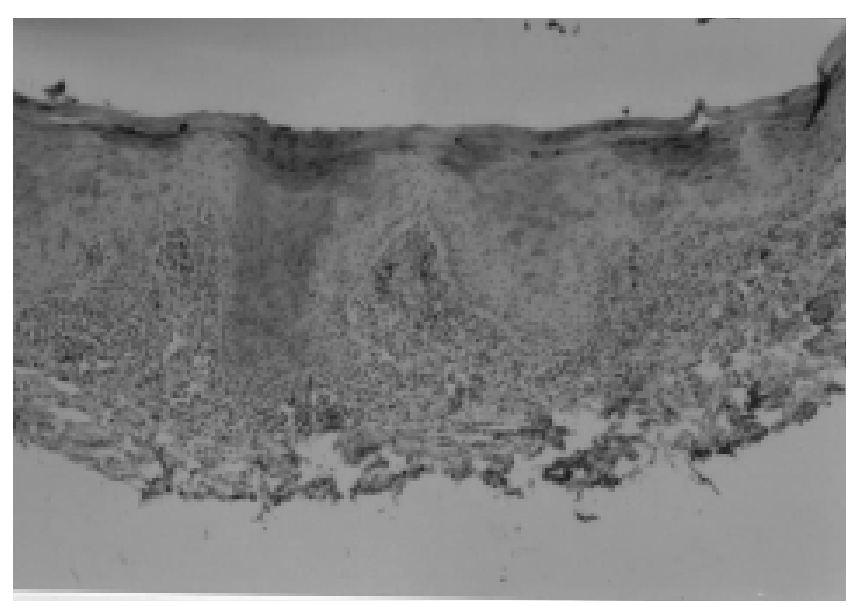

Fig. 9 CK 13: delay in the expression, with similar aspect to that described in the previous photo, although greater intensity of the reaction in the upper portions of the Malpighian layer is evident. Obj: $\times 10$ 


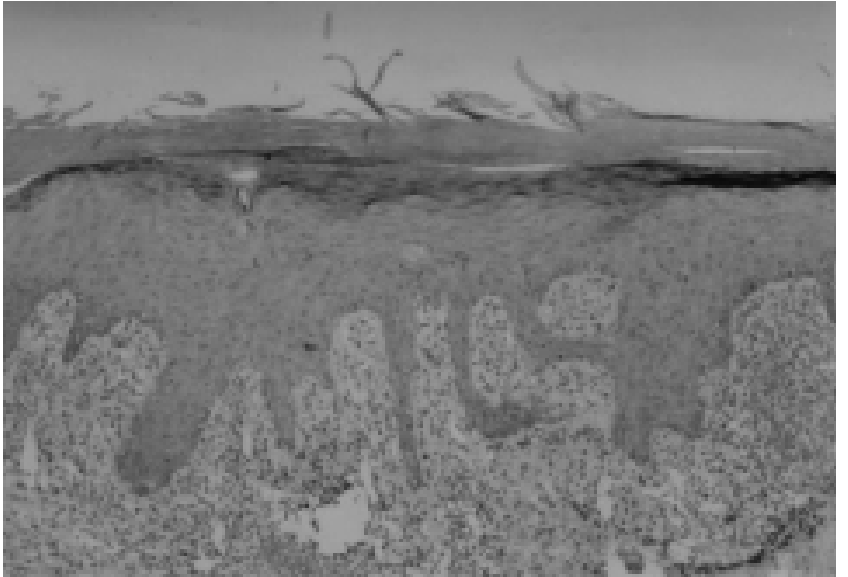

Fig. 10 CK 14: area with greater inflammation and squamous hyperplasia, with marking on all layers of the epithelium. Obj: $\times 10$

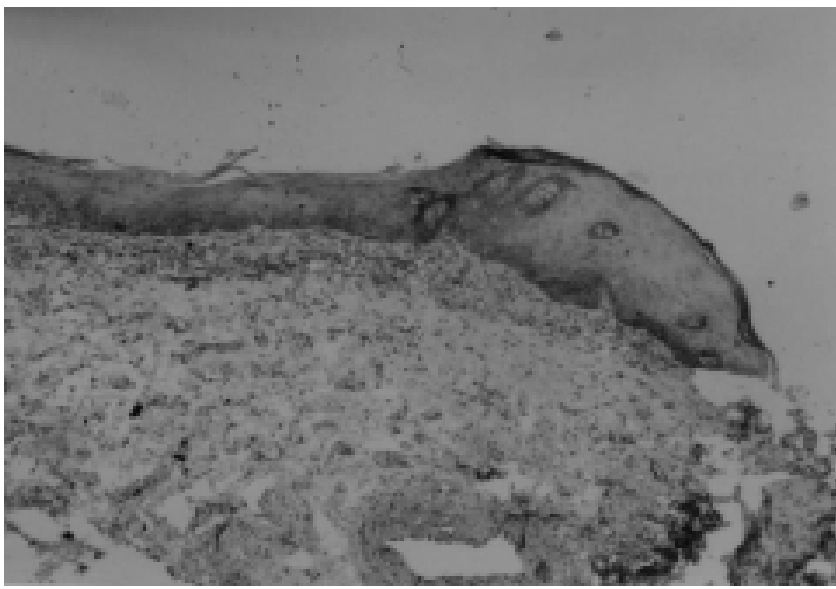

Fig. $11 \mathrm{CK}$ 14: basal and parabasal expression in area with scarce inflammation. Obj: $\times 10$

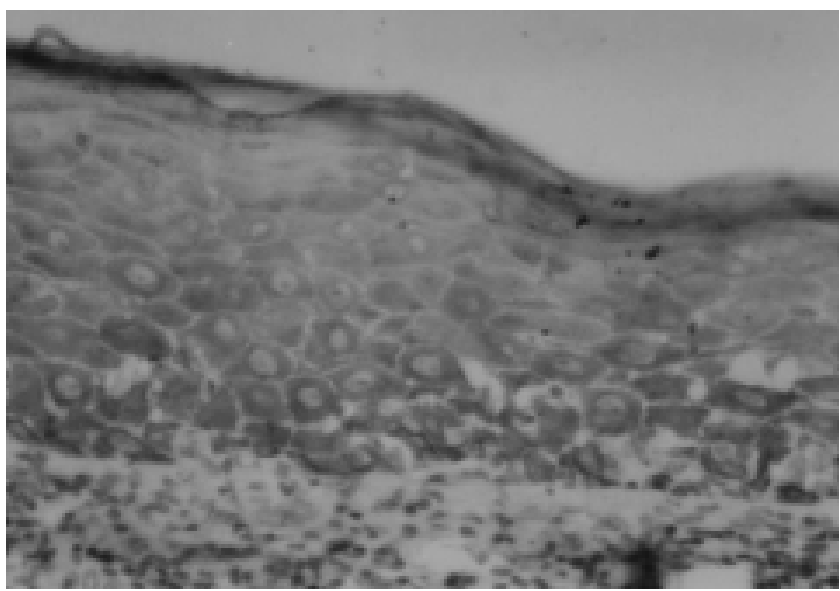

Fig. 12 CK 14: marking on all layers of the epithelium. Obj.: $\times 40$

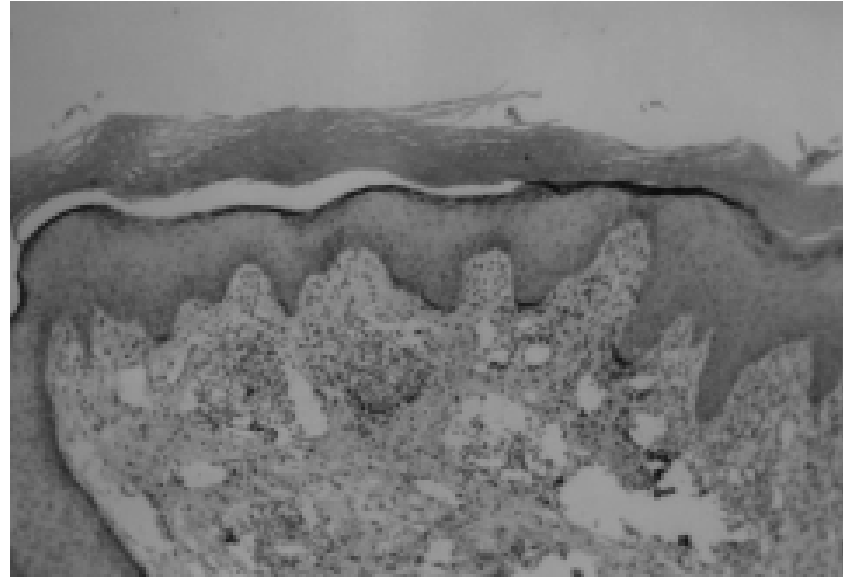

Fig. 13CK 14: area with scarce inflammation, with predominantly basal expression. Obj.: $\times 10$

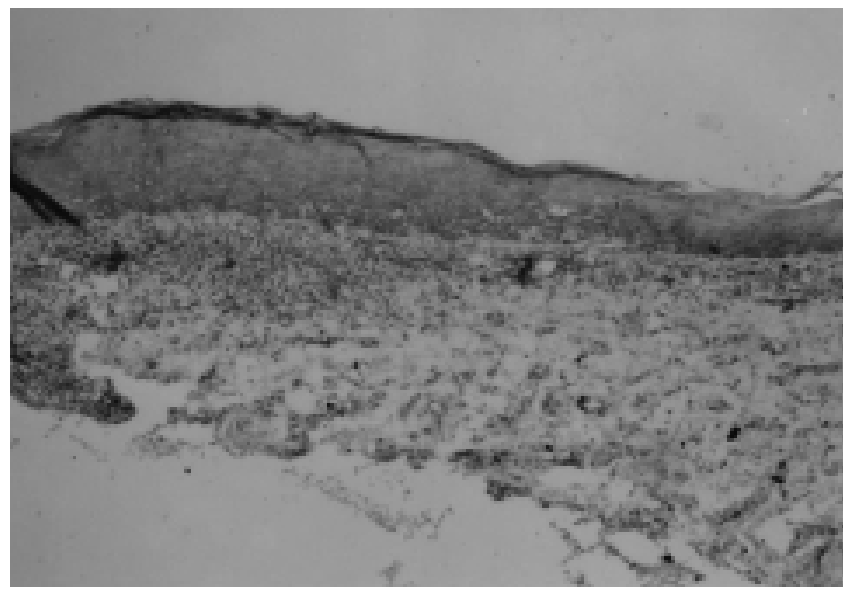

Fig. 14 CK 14: expression on all layers of the epithelium, above band-like inflammatory infiltrate. Obj.: $\times 10$

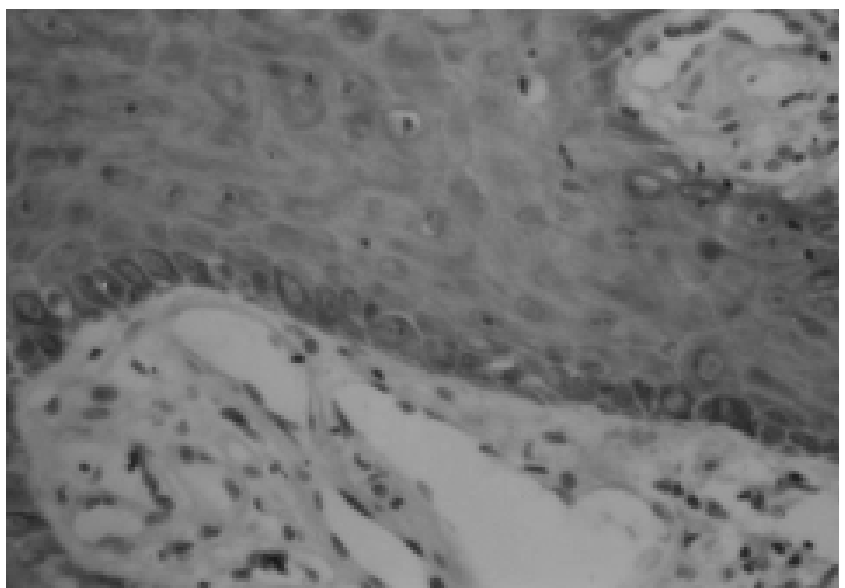

Fig. 15 CK 14: basal and suprabasal marking. Obj.: $\times 40$ 


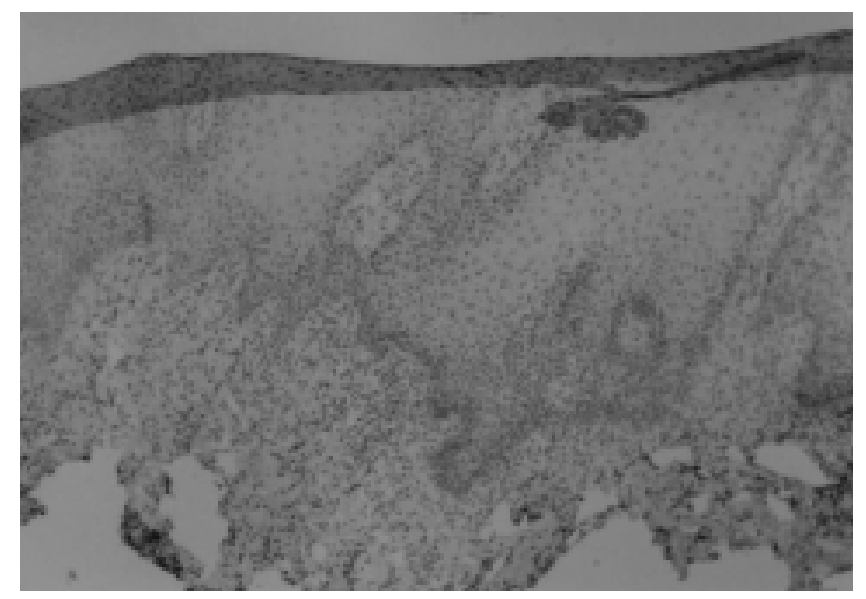

Fig. 16CK 19: focal basal positivity. Obj: $\times 10$

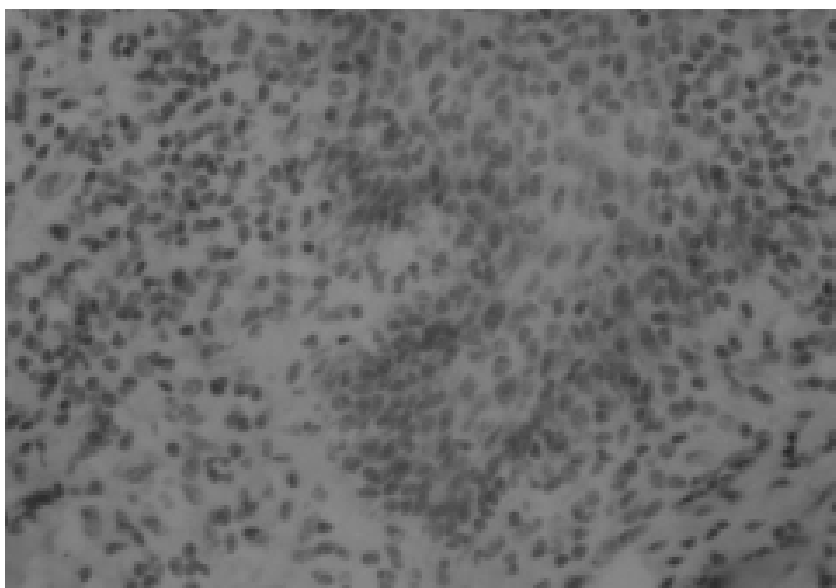

Fig. 17 CK 19: focal basal positivity (detail of previous photo). Obj.: $\times 40$

\section{Discussion}

The study of CKs in OLP may help to improve our understanding of the disease, by providing references that aid in its correct diagnosis in face of other pathological conditions that present clinical and/or histopathological similarities, some of them with potential for malignant transformation, including OLP itself.

In the present study, an analysis of the expression of CKs $10,13,14$, and 19 in OLP lesions was made, comparing them with the findings in literature regarding the normal oral mucosa and OLP. These four CKs were chosen as they are frequently expressed in the normal oral mucosa. As seen in Table 4, the expression of CKs by the epithelium of the oral mucosa in keratinized areas (lips, gingiva, back of tongue and hard palate) was quite different from that

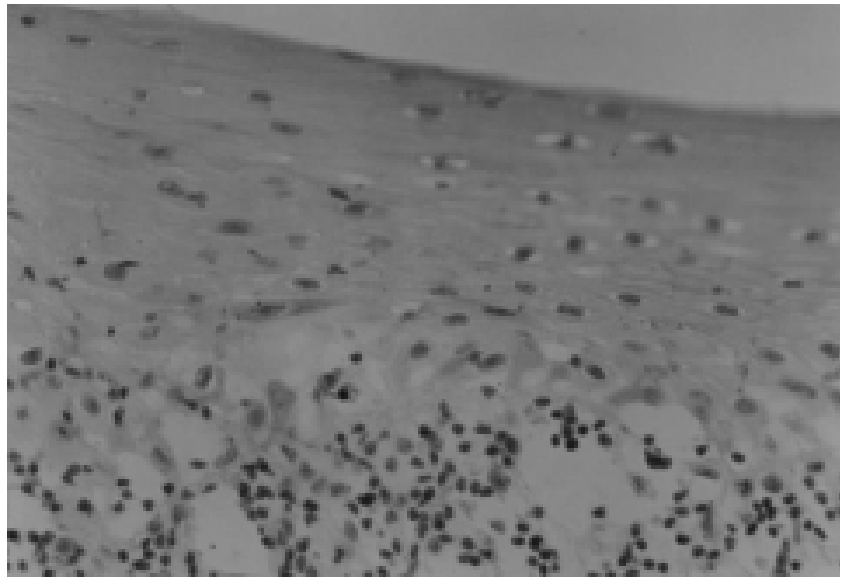

Fig. 18 CK 19: reaction positivity in some basal and parabasal cells. Obj: $\times 10$

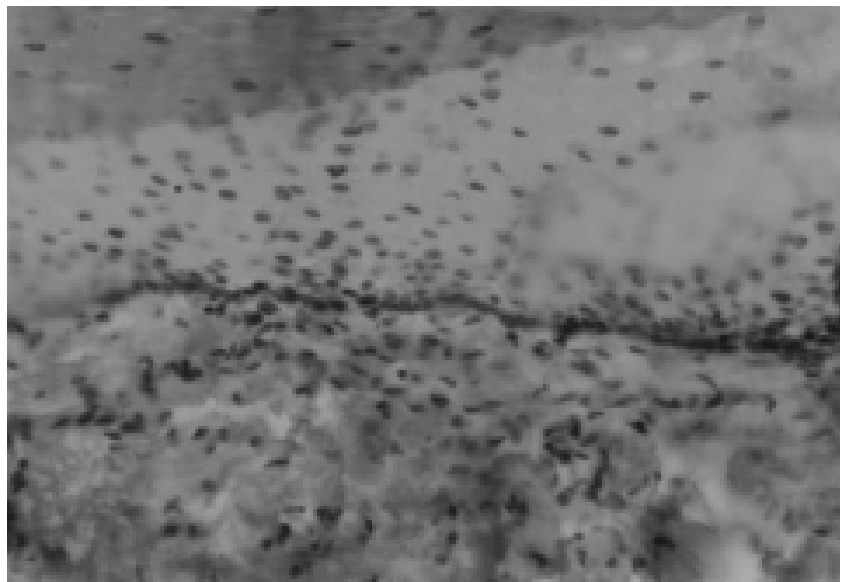

Fig. 19 CK 19: positive marking along the basal layer. Obj: $\times 10$

in the non-keratinized areas (lip and buccal mucosa, ventral surface of the tongue and soft palate).

\section{Dysplastic alterations}

In the present study, it was observed that $42.3 \%$ of the samples presented dysplasia, all of mild degree. This was higher than the results obtained by De Jong (1984) and Caffarena (1986), who found dysplastic alterations in $25 \%$ and $26.1 \%$, respectively $(12,13)$.

Studying 100 patients with OLP, Odukoya found mild dysplasia in 57\%, moderate in $9 \%$ and severe in $2 \%$ (14). These findings were different from the present study, not only by the greater percentage of dysplasia found, but also by the observation of cases presenting dysplasia in moderate and severe grades.

It must be emphasized that the presence of mild grade 
dysplasia is a common finding in OLP, and does not indicate a possibility for malignant transformation of the epithelium $(12,15)$.

\section{Cytokeratins \\ Cytokeratin 10}

CK 10 is considered a specific marker of terminal differentiation of the keratinocytes, as it is frequently found in stratified keratinized epithelia. In the oral mucosa it is found in the keratinized areas, always presenting a suprabasal marking pattern, while in the non-keratinized areas, its expression is essentially negative (15-18).

In the present study, suprabasal marking for CK 10 was found in 16 of the 17 studied samples, with focal characteristics in 14 of the 16 positive samples. The only sample presenting negative suprabasal marking for CK 10, belonged to a patient presenting plaque-type OLP in the buccal mucosa, with no apparent reason for the isolated observation. Along the basal layer, all of the samples presented negative marking, as expected. No difference in marking was observed between the keratinized and nonkeratinized mucosa samples. These results showed that, in presence of lichen planus, the non-keratinized mucosa presented suprabasal expression of CK 10, which does not happen when in normal state; while the keratinized mucosa, that usually expresses CK 10 in a diffused manner, underwent a strong reduction of that marking in most of the samples. Those findings are in line with the results obtained by Maeda, Boisnic, Van der Velden and Bloor $(16,17,19,20)$. In his comparative study of 13 cases of normal buccal mucosa with 17 cases of OLP, Maeda et al. used polyclonal antibodies AE1 (for CKs 10, 13, 14, 15, 16 and 19) and AE2 (for CKs 1, 2 and 10). Besides monoclonal antibodies for CKs 5, 8, 13 and 19 (16); they studied CKs 4, 5, 10, 11, 13, 14, 17 and 19, in 20 samples of normal oral keratinized and non-keratinized mucosa, and samples of OLP in keratinized and non-keratinized mucosa, 5 cases of each (17). Van der Velden's work included 18 samples of varied benign lesions of the oral mucosa, with 3 of OLP. 14 different monoclonal antibodies, markers of CKs were used (19). Bloor evaluated CKs 1 , 4, 10 and 13 in 10 samples of non-dysplastic oral keratosis, 15 of OLP and 3 of lichenoid reaction (20). These authors considered that the presence of hyperkeratosis or parakeratosis in the lesions of OLP could be responsible for the above findings. This would demonstrate the alterations in the differentiation program of the cells in both epithelia, whether keratinized or not. However, they emphasized that CK 10 should not be considered a marker for OLP, but as a marker for presence of keratinization.

Studying 20 samples of OLP lesions, Chaiyarit found significant markings for CK 10, both in the basal layer and in the suprabasal portion of the epithelium, differing from the results observed in the present study, and from the findings of the previously mentioned studies. The author attributes such unprecedented basal marking to the possible action of cytokines secreted by the T-lymphocytes present in the inflammatory cell infiltrate, on the expression of $\mathrm{CK}$ 10 (21). By comparing normal gingiva with inflamed gingiva, Ouhayon et al. and Mackenzie et al. described alterations in the expression of the CK, which were inversely proportional to the intensity of the inflammatory cell infiltrate $(22,23)$. However, there was no opportunity to observe such findings in the present study.

\section{Cytokeratin 13}

CK 13 is one of the main components of most of the stratified non-keratinized squamous epithelia $(18,24)$. In the non-keratinized oral mucosa, CK 13 is usually expressed in a homogeneous pattern, restricted to the suprabasal portions of the epithelium, while in the keratinized areas it presents essentially negative markings $(16,17,19)$.

In the present study, negative suprabasal markings were observed in 5 of the 23 samples and, in the 18 cases where positivity was observed, 16 presented altered markings by presence of delay. Besides, all of the samples presented negativity for the markings along the basal layer. Those results showed that, in the presence of OLP, the nonkeratinized mucosa presented reduction in the pattern and heterogeneity in the marking for $\mathrm{CK} 13$, in relation to the normal mucosa. Additionally, even in the samples in which CK 13 was expressed, alteration to the normal process of cellular differentiation occurred in most cases, translated by the presence of delay in the marking. Differences were not observed in the marking pattern between keratinized and non-keratinized mucosa. These findings were in line with the results obtained by Maeda, Boisnic, Van der Velden and Bloor $(16,17,19,20)$. Maeda et al.reported reduction or negativity in the marking for CK 13 in over half of the 17 cases of OLP in non-keratinized mucosa studied (16). They suggested that the observed alterations were not influenced by the type of keratinization, in line with the present study. Boisnic reported reduction or negativity in the marking for CK 13 in the 10 cases of OLP studied, with five in keratinized mucosa and five in nonkeratinized mucosa, while Van der Velden obtained the same result in his three cases $(17,19)$. Bloor et al. obtained reduction or absence of marking for CK 13 in the parabasal area of the epithelium, a result also observed in the present study (20). Those authors state that these alterations occurred by influence of the presence of inflammatory cell infiltrate, particularly lymphocytes, by release of cytokines. 


\section{Cytokeratin 14}

In the normal epithelia, CK 14 is almost exclusively expressed in the basal layer, with presence both in the simple, as well as the stratified epithelia. In the oral mucosa, this CK can be found in the basal cells, both in the keratinized, as well as the non-keratinized areas $(16,17,24,25)$. Due to its location, CKs 5 and 14 are considered as those mostly responsible for the maintenance of the integrity of the basal layer, being the main components of the hemidesmosomes. The expression of CK 5 and CK 14 defective genes imposes intense formation of bullae in the skin and in the oral mucosa of transgenic mice, representing a phenotype similar to epidermolysis bullosa simplex (26).

In a study with humans, including families of bearers of that disease, local mutations were demonstrated in the extremities of the helical areas of the molecules of both $\mathrm{CKs}$, with mutations identified in the carboxy- and aminoterminal areas. These mutations are expressed in the epidermal basal layer, weakening its structure and allowing slight mechanical trauma to cause its rupture, leading to the formation of bullae (27).

The results of the present study showed that, in OLP, an alteration occurred in the marking pattern in relation to the normal mucosa, with CK 14 being expressed along all the layers of the epithelium, instead of remaining limited to the basal layer. Taking these findings into account, and the previously mentioned study by Mackenzie on inflamed gingiva, where the author attributes the suprabasal expression of CK 14 to the presence of the inflammatory cell infiltrate, it was possible to conclude that the inflammation interferes in the process of cell differentiation of the oral mucosa, leading to the abovedescribed alterations (23).

However, the results observed in the present study showed differences in relation to those obtained by Boisnic, who found positive marking for CK 14 limited to few cells of the basal layer, and negative marking in the suprabasal portion of the epithelium (17). The author suggests the necrotic alterations of the basal keratinocytes as a probable cause for these findings.

On the other hand, studying benign lesions of the oral non-keratinized mucosa, Van der Velden described positive marking for CK 14 in a homogeneous manner in the basal layer, and focal marking in the suprabasal portion (19). His sample included three cases of OLP, besides other 15 fibroma lesions and other unspecific hyperkeratotic and/or hyperplastic lesions. The author speculated that these alterations were probably linked to the presence of the inflammatory process that accompanies those lesions.

The presence of positive marking for CK 14 along all of the dysplastic epithelial layers was reported in the studies published by Heyden and Su $(28,29)$. They considered these alterations as a probable sign of malignant transformation. In the present study, similar results were not observed. The presence of positive marking along all the layers of the epithelium occurred in all of the samples, regardless of the presence or absence of dysplasia. In line with these findings, it was not possible to consider the suprabasal marking separately for CK 14 as a marker of dysplastic alterations or of a tendency to malignant transformation of the tissue.

\section{Cytokeratin 19}

CK 19 is normally present in the simple epithelia, and can eventually be also expressed in the stratified nonkeratinized epithelia $(24,30)$. According to the literature, CK 19 displays some variations as to its expression pattern in the normal non-keratinized oral mucosa. Van der Velden reported that it is expressed heterogeneously in the basal layer, and can present eventual focuses in the suprabasal portion of the epithelium (19). Maeda found homogeneous positive basal marking in 6 cases, focal in four, and negative basal marking in three cases studied (16). Boisnic and Lindberg described positive marking in some cells of the basal layer $(17,31)$. In relation to the normal keratinized mucosa, all the abovementioned authors unanimously stated that marking is always negative for CK 19 $(16,17,19,31)$.

In the present study, the suprabasal portion of the epithelium showed negative marking in all 21 samples, while, in the basal layer, only four samples presented positive marking for this $\mathrm{CK}$, all of focal and quite discrete character. Three of those were located in the buccal mucosa and one in the gingiva. It was observed that OLP lesions presented a marking pattern for CK 19 similar to that found in literature in relation to the normal mucosa, except for one sample of keratinized mucosa, which presented positive marking. In spite of the isolated case, this positivity demonstrated that the keratinized epithelium could express CK 19 eventually during progression of the disease.

By comparing the expression of CK 19 in the lesions of OLP from the literature with the results obtained in the present study, similarity to those reported by Van der Velden was found, who observed positive basal focal marking and negative suprabasal marking in three cases of OLP (19). He reported that the positivity of the marking occurred only in cases of presence of inflammatory cell infiltrate.

On the other hand, Boisnic reported positive marking in all cells of the basal layer and also positive focal marking in the suprabasal portion of the epithelium, in lichen planus 
of the non-keratinized mucosa (17). Additionally, she observed basal focal positive marking in 5 cases of lichen planus of the gingival and speculated the possibility of suprabasal expression of CK 19 to be considered as a premalignant marker in the lesions of OLP.

Several studies investigated the association between the expression of this $\mathrm{CK}$ and lesions with dysplastic alterations and carcinomas, considering them as a marker of malignant alteration of the epithelium (31-34). Lindberg found positive basal and suprabasal marking in all of the studied cases of oral lesions that presented moderate to severe dysplasia and in the in situ carcinomas, regardless of their location or presence of hyperkeratosis, which was different from the marking pattern in the normal mucosa, as mentioned previously (31). In the cases of lesions presenting mild dysplasia, the marking shown was variable, with some cases of suprabasal positivity. In the abovementioned study, the author suggested that the suprabasal expression of CK 19 could be considered as a marker of dysplastic alteration in pre-malignant lesions.

Schulz found expression of CK 19 in OLP in a pattern similar to that of normal non-keratinized mucosa, in line with the findings obtained in the present study (25). He also observed emergence of the expression of this $\mathrm{CK}$, in some cases of squamous cell carcinoma and leukoplakia.

Differently, Su, studying the expression of CK 19 in the normal oral mucosa, dysplastic lesions and carcinomas, observed a variable marking pattern (29). The author concluded that, in line with those findings, one could not consider the suprabasal expression of CK 19 as a potential marker for malignant transformation of the epithelium.

In the present study, the marking pattern for CK 19 did not show alteration due to the presence of dysplastic alterations in the epithelium. It should be mentioned, however, that in all samples studied, these dysplastic alterations were considered to be of light grade, which probably contributed to these findings. As already mentioned previously, De Jong and Urbizo-Vélez reported that the oral lesions of the lichen planus present mild dysplastic alterations with relative frequency, not exhibiting a trend for malignant transformation $(12,15)$. Future studies in OLP with moderate to severe dysplasia should be conducted for better evaluation of the possible role of CK 19 as a trend marker of the epithelium for malignant transformation.

It is also believed that, based on the observation that the pattern of expression of these CKs in OLP presents variations from one population studied to another, combined with the fact that OLP is a relatively rare disease, subsequent studies may become necessary seeking associations with clinical-histopathological factors, including different population groups and/or other CKs not studied.

According to the results obtained, it can be concluded that the pattern of expression of CKs 10,13 and 14 is altered in the oral cavity, during the disease activity of lichen planus. The inflammatory process and hyperkeratosis or parakeratosis seem to have interfered with the expression of those CKs.

CK 19 did not present significant differences in its expression pattern.

It was observed that, in the sample studied, the presence of mild dysplasia did not alter the expression of CKs in issue. The suprabasal expression of CKs 14 and 19 cannot be considered as a marker of mild dysplastic alterations in the epithelium.

\section{References}

1. Jacques C, de Aquino AM, Ramos-e-Silva M (2005) Cytokeratins and dermatology. Skinmed 4, 354360.

2. Pereira ALC, Jacques CMC, Cabral MG, Cardoso AS, Ramos-e-Silva M (2004) Oral lichen planus. Part II: therapy and malignant transformation. Skinmed 3, 19-22.

3. Silverman S, Bahl S (1997) Oral lichen planus update: clinical characteristics, treatment responses and malignant transformation. Am J Dent 10, 259263.

4. Fujii H, Ohashi M, Nagura H (1988) Immunohistochemical analysis of oral lichen planuslike eruption in graft-versus-host disease after allogeneic bone marrow transplantation. Am J Clin Pathol 89, 177-186.

5. Mattsson T, Sundqvist KG, Heimdahl A, Dahllöf G, Ljungman P, Ringdén O (1992) A comparative immunological analysis of the oral mucosa in chronic graft-versus-host disease and oral lichen planus. Arch Oral Biol 37, 539-547.

6. Jacques CMC, Pereira ALC, Cabral MG, Cardoso AS, Ramos-e-Silva M (2003) Oral lichen planus. Part I: epidemiology, clinics, etiology, immunopathogeny, and diagnosis. Skinmed 2, 342-347.

7. Ishida-Yamamoto A, Takahashi H, Iizuka H (2002) Lessons from disorders of epidermal differentiationassociated keratins. Histol Histopathol 17, 331-338.

8. Oshima RG (2002) Apoptosis and keratin intermediate filaments. Cell Death Differ 9, 486-492.

9. Lane EB 1982 Monoclonal antibodies provide specific intramolecular markers for the study of epithelial tonofilament organization. J Cell Biol 92, 665-673.

10. Kramer IR, Lucas RB, Pindborg JJ, Sobin LH. 
(1978) Definition of leukoplakia and related lesions: an aid to studies on oral precancer. Oral Surg Oral Med Oral Pathol 46, 518-539.

11. Bánóczy J, Csiba A (1976) Occurrence of epithelial dysplasia in oral leukoplakia. Analysis and followup study of 12 cases. Oral Surg Oral Med Oral Pathol 42, 766-774.

12. De Jong WF, Albrecht M, Bánóczy J, van der Waal I (1984) Epithelial dysplasia in oral lichen planus. A preliminary report of a Dutch-Hungarian study of 100 cases. Int J Oral Surg 13, 221-225.

13. Caffarena MP (1986) Displasia epitelial en el liquen plano bucal. An Fac Odont 24, 19-26.

14. Odukoya O, Gallagher G, Shklar G (1985) A histologic study of epithelial dysplasia in oral lichen planus. Arch Dermatol 121, 1132-1136.

15. Urbizo-Vélez J, Rodriguez Pérez I, Albrecht M, Bánóczy J (1990) Comparative histopathological studies in oral lichen planus. Acta Morphol Hung 38, 71-81.

16. Maeda H, Reibel J, Holmstrup P (1994) Keratin staining pattern in clinically normal and diseased oral mucosa of lichen planus patients. Scand J Dent Res, 102, 210-215.

17. Boisnic S, Ouhayoun JP, Branchet MC, Frances C, Béranger JY, Le Charpentier Y, Szpirglas H (1995) Alteration of cytokeratin expression in the oral lichen planus. Oral Surg Oral Med Oral Pathol Oral Radiol Endod 79, 207-215.

18. Moll R, Franke WW, Schiller DL, Geiger B, Krepler R (1982) The catalog of human cytokeratins: patterns of expression in normal epithelia, tumors and cultured cells. Cell 31, 11-24.

19. Van der Velden LA, Manni JJ, Ramaekers FC, Kuijpers W (1999) Expression of intermediate filament proteins in benign lesions of the oral mucosa. Eur Arch Otorhinolaryngol 256, 514-519.

20. Bloor BK, Seddon SV, Morgan PR (2000) Gene expression of differentiation-specific keratins (K4, $\mathrm{K} 13$, K1 and K10) in oral non-dysplastic keratoses and lichen planus. J Oral Pathol Med 29, 376-384.

21. Chaiyarit P, Kafrawy AH, Miles DA, Zunt SL, Van Dis ML, Gregory RL (1999) Oral lichen planus: an imunohistochemical study of heat shock proteins (HSPs) and cytokeratins (CKs) and a unifying hypothesis of pathogenesis. J Oral Pathol Med 28, 210-215.

22. Ouhayoun JP, Goffaux JC, Sawaf MH, Shabana AH, Collin C, Forest N (1990) Changes in cytokeratin expression in gingiva during inflammation. J Periodontal Res 25, 283-292.

23. Mackenzie IC, Gao Z (1993) Patterns of cytokeratin expression in the epithelia of inflamed human gingiva and periodontal pockets. J Periodontal Res 28, 4959.

24. Chu PG, Weiss LM (2002) Keratin expression in human tissues and neoplasms. Histopathology 40, 403-439.

25. Schulz J, Ermich T, Kasper M, Raabe G, Schumann D (1992) Cytokeratin pattern of clinically intact and pathologically changed oral mucosa. Int J Oral Maxillofac Surg 21, 35-39.

26. Coulombe PA, Omary MB (2002) "Hard" and "soft" principles defining the structure, function and regulation of keratin intermediate filaments. Curr Opin Cell Biol 14, 110-122.

27. Rao KS, Babu KKR, Gupta PD (1996) Keratins and skin disorders. Cell Biol Int 20, 261-274.

28. Heyden A, Huitfeldt HS, Koppang HS, Thrane PS, Bryne M Brandtzaeg P (1992) Cytokeratins as epithelial differentiation markers in premalignant and malignant oral lesions. J Oral Pathol Med 21, 7-11.

29. Su L, Morgan PR, Lane EB (1996) Keratin 14 and 19 expression in normal, dysplastic and malignant oral epithelia. A study using in situ hybridization and immunohistochemistry. J Oral Pathol Med 25, 293-301.

30. Stasiak PC, Purkis, PE, Leigh IM, Lane EB (1989) Keratin 19: predicted amino acid sequence and broad tissue distribution suggest it evolved from keratinocyte keratins. J Invest Dermatol 92, 707-716.

31. Lindberg K, Rheinwald JG (1989) Suprabasal 40kd keratin (K19) expression as an immunohistologic marker of premalignancy in oral epithelium. Am J Pathol 134, 89-98.

32. Wu YJ, Rheinwald JG (1981) A new small (40 kd) keratin filament protein made by some cultured human squamous cell carcinomas. Cell 25, 627635.

33. Bártek J, Bártková J, Schneider J, TaylorPapadimitriou J, Kovarík J, Rejthar A (1986) Expression of monoclonal antibody-defined epitopes of keratin 19 in human tumours and cultured cells. Eur J Cancer Clin Oncol 22, 1441-1452.

34. Cintorino M, Petracca R, Vindigni C, Tripodi SA, Leoncini P (1990) Topography-related expression of individual cytokeratins in normal and pathological (non-neoplastic and neoplastic) human oral mucosa. Virchows Arch A Pathol Anat Histopathol 417, 419-426. 